Research Paper

\title{
Carotid Intima-media thickness in childhood and adolescent obesity rela- tions to abdominal obesity, high triglyceride level and insulin resistance
}

\author{
Jie Fang ${ }^{\bowtie}$, Jian Ping Zhang, Cai Xia Luo, Xiao Mei Yu, Lan Qiu Lv \\ Department of Endocrinology, Ningbo Women and Children's Hospital, Ningbo, 315000, China
}

$\triangle$ Corresponding author: Jie Fang, Department of Endocrinology, Ningbo Women and Children's Hospital, Ningbo, 315000, China. Tel: +86-13957882013; E-mail: fangjie121108@yahoo.com.cn

Received: 2010.04.13; Accepted: 2010.08.08; Published: 2010.08.18

\begin{abstract}
Aim: To investigate risk factors which impact on common carotid artery intima media thickness (IMT).

Methods: A total of 86 obese children and adolescents and 22 healthy children and adolescents with normal weight were enrolled. Moreover, 23 of 86 obese children and adolescents were diagnosed with metabolic syndrome (MetS). The clinical, biochemical data and the IMT of the common carotid artery were measured in all subjects.

Results: Obese and obese with MetS subjects demonstrated a significantly $(p<0.01)$ thicker intima media $(0.69 \mathrm{~mm}, 0.66 \mathrm{~mm})$ as compared to the control group $(0.38 \mathrm{~mm})$, but there was no significant difference of IMT between obese and MetS group. IMT was correlated to body weight, body mass index, waist circumference, waist to hip ratio, systolic blood pressure, diastolic blood pressure, fasting insulin, homoeostasis model assessment-insulin resistance, triglyceride, high-density lipoprotein- cholesterol, low-density lipoprotein-cholesterol, alanine aminotransferase, aspartate aminotransferase and fatty liver. Waist circumference, waist to hip ratio, triglyceride and homoeostasis model assessment-insulin resistance were independent determinants of mean IMT level.

Conclusion: Obesity especially abdominal obesity, high TG and insulin resistance may be the main risk predictors of increased IMT.
\end{abstract}

Key words: obesity, metabolic syndrome, intima-media thickness, children, adolescents

\section{Introduction}

The rapidly increasing prevalence of obesity among children is one of the most challenging problems. The prevalence of the metabolic syndrome (MetS) in children is increasing exponentially because of global increase in obesity. As indicated in previous studies [1,2,3], children and adolescents with risk factors such as obesity, dyslipidemia, elevated blood pressure and impaired glucose metabolism are at increased risk of developing atherosclerosis in adulthood. It has been found that obesity results in the early onset of adulthood chronic disease such as cardio-cerebrovascular disease. Recent researches $[4,5,6]$ have revealed that adiposity-associated inflammatory factors such as C-reactive protein (CRP), interleukin (IL)-6 and tumor necrosis factor (TNF)- $a$ may play a role in promoting adverse vascular outcomes.

The intima media thickness (IMT) of the common carotid artery (CCA) is a well-known marker of subclinical atherosclerosis and is a noninvasive, feasible, reliable and inexpensive method for detecting development of subclinical atherosclerosis. Studies in adults have revealed that IMT was related to cardiovascular risk factors and could predict the possibility of future cardio-cerebrovascular disease $[7,8]$. Increase 
IMT was also reported in children with obesity, familial hypercholesterolemia and nonalcoholic fatty liver disease (NAFLD) compared with control children.

There has been no statistical data about the association between IMT and the components of MetS since new definition for children and adolescent MetS was published by International Diabetes Federation (IDF). This study aimed to verify the relationships among obesity, dyslipidemia, elevated blood pressure, impaired glucose metabolism, chronic inflammation, fatty liver and IMT to explore as to which of these factors are related to IMT.

\section{Subjects and Methods}

\section{Subjects}

A total of 86 obese Chinese children were enrolled from July 2008 to March 2009. The obese group was defined as obese children without MetS, which included 46 boys and 17 girls with a mean age of $10.5 \pm 1.6$ years (range 7.4 to 13.3 years). The MetS group was defined as obese children with MetS, which included 18 boys and 5 girls with a mean age of $10.9 \pm 1.6$ years (range 7.6 to 14.2 years). Children with other chronic disease (endocrine disease, hereditary disease, or systemic inflammation) or those taking any medications were excluded. The control group consisted of 22 healthy non-obese children, which included 16 boys and 6 girls with a mean age of $11.1 \pm$ 2.1 years (ranging from 7.6 to 14.8 years).

Consent was obtained from the parents and the Ethical Committee of the Children's Hospital of Zhejiang University School of Medicine.

\section{Diagnostic Criteria}

Obesity was defined as body mass index (BMI) $\geq 95^{\text {th }}$ percentile using the childhood date of Working Group on Obesity in China (WGOC) [9]. According to the IDF criteria for children and adolescents [10], MetS was identified if a subject had increased waist circumference ( $>$ 90th percentile) [11] and also had $\geq$ 2 of the following: 1) impaired fasting blood glucose ( $\geq 5.6 \mathrm{mmol} / \mathrm{L}$ ), or Type 2 Diabetes Mellitus; 2) increased blood pressure ( $\geq 130 \mathrm{mmHg}$ systolic and/or $\geq 85 \mathrm{mmHg}$ diastolic ); 3) elevated plasma triglycerides ( $\geq 1.7 \mathrm{mmol} / \mathrm{L}$ ); 4) high plasma high-density lipoprotein cholesterol ( $<1.03 \mathrm{mmol} / \mathrm{L})$.

\section{Clinical characteristics}

The body weight was assessed using a calibrated standard balance beam, height was measured by a standard height bar, and BMI was calculated as body weight $(\mathrm{kg})$ divided by square height $\left(\mathrm{m}^{2}\right)$. Waist circumference (WC) was measured at the midway be- tween the lower rib and the iliac crest, hip circumference was measured at the widest part at the gluteal region. Systolic blood pressure (SBP) and diastolic blood pressure (DBP) were measured twice at the right arm after a 10-minute rest in the supine position using an automated sphygmomanometer.

\section{Biochemical measurements}

Samples were drawn between 8 and 9 am after fasting for 10 hours. Triglycerides (TG), total cholesterol (TC) were measured by enzymatic and cholesterol oxidase method respectively, high plasma high-density lipoprotein cholesterol (HDL-C) and low-density lipoprotein cholesterol (LDL-C) were both detected by the direct assay method, alanine aminotransferase (ALT) and aspartate aminotransferase (AST) were tested by enzyme-linked immunosorbent assay method. Fasting plasma glucose (FPG) was measured by glucose oxidase method; fasting plasma insulin (FINS) was measured by radioimmunity assay (Modula Analytics PP, Roche). Both intra-assay and inter-assay coefficient of variations were less than $2.1 \%$ and $4.4 \%$, respectively. Plasma levels of IL-6 and TNF were measured by enzyme-linked immunosorbent assay method (Ju Ying bioscitech, Shenzhen, China), with both intra-assay and inter-assay coefficient of variations being less than $10 \%$.

\section{IMT measurement}

IMT was measured by B-mode ultrasound using a $10-\mathrm{MHz}$ linear transducer (Philips HD7). The subjects were examined supine with the neck extended and the probe in the antero-lateral position. All measurements of IMT were made in the longitudinal plane at the point of maximum thickness on the far wall of the common carotid artery along a $1 \mathrm{~cm} \mathrm{sec-}$ tion of the artery proximal to the carotid bulb. The IMT was defined as the distance between the intimia-blood interface and the adventitia-media junction. After freezing the image, the measurements were made using electronic calipers. The maximal thicknesses of the intima-media width were measured to give three readings and the mean value was used for statistical purposes.

\section{Statistical analysis}

Statistical analysis was performed with SPSS 13.0. WHR, FBG, HOMA-IR, TNF were normalized by log-transformation. Statistically significant differences were tested for qualitative items by $\chi^{2}$ test and for quantitative items by One-Way ANOVA. Thereafter, associations were examined by Pearson correlation analysis for continuous variables, and by Spearman correlation analysis for categorical variables. Finally, 
multiple stepwise linear regression analysis was used to examine relationships between mean IMT and all other variables investigated. A $p<0.05$ was considered statistically significant.

\section{Results}

\section{The characteristics of three groups}

The obese and MetS group both demonstrated increased mean IMT, body weight, BMI, WC, WHR, SBP, FINS, HOMA-IR, Ig (HOMA-IR), TG, LDL-C, ALT and AST levels, decreased HDL-C levels and higher prevalence of fatty liver $(p<0.05)$. Furthermore, the MetS group showed higher DBP compared with the control group. The children of MetS group had higher values of WC, SBP and TG, and lower HDL-C than these of obese group. There was no sta- tistical difference in the age and sex among three groups ( $p=0.400,0.672$ ), as shown in table 1 .

\section{The relationship between IMT and all other va- riables investigated}

In all subjects, mean IMT of CCA was significantly related to body weight, BMI, WC, $\lg$ (WHR), SBP, DBP, FINS, $\lg$ (HOMA-IR), TG, HDL-C, LDL-C, ALT, AST and fatty liver, as shown in table 2. IMT was not significantly related to age, sex, FBG, TC, IL-6 and $\lg$ (TNF).

Finally, the multiple stepwise linear regression analysis showed that WC, $\lg$ (WHR), TG, lg (HOMA-IR) were independent determinants of mean IMT level. All the other factors were excluded in the equations, as shown in table 3.

Table I The characteristics of obese, MetS and control groups

\begin{tabular}{|c|c|c|c|c|c|}
\hline & MetS group & Obese group & Control group & $F / \chi^{2}$ & $P$ \\
\hline Age(y) & $10.9 \pm 1.6$ & $10.5 \pm 1.6$ & $11.1 \pm 2.1$ & 0.924 & 0.400 \\
\hline $\operatorname{Sex}(\mathrm{F} / \mathrm{M})$ & $18 / 5$ & $46 / 17$ & $16 / 6$ & 0.425 & 0.672 \\
\hline Weight(kg) & $70.15^{* *}$ & $60.65^{* *}$ & 31.86 & 96.045 & $<0.001$ \\
\hline $\operatorname{BMI}\left(\mathrm{kg} / \mathrm{m}^{2}\right)$ & $29.63^{* *}$ & $28.04^{* *}$ & 17.32 & 182.510 & $<0.001$ \\
\hline $\mathrm{WC}(\mathrm{cm})$ & $94.22^{* \star \#}$ & $88.83^{* *}$ & 58.83 & 94.835 & $<0.001$ \\
\hline WHR & $0.96^{* *}$ & $0.96^{* *}$ & 0.76 & 46.027 & $<0.001$ \\
\hline $\lg (\mathrm{WHR})$ & $-0.02^{* *}$ & $-0.02^{* *}$ & -0.12 & 40.424 & $<0.001$ \\
\hline $\mathrm{SBP}(\mathrm{mmHg})$ & $122.3^{* * \#}$ & $111.67^{* *}$ & 103.32 & 15.079 & $<0.001$ \\
\hline $\mathrm{DBP}(\mathrm{mmHg})$ & $70.91^{\text {*k }}$ & 67.06 & 63.14 & 6.994 & 0.002 \\
\hline $\mathrm{FBG}(\mathrm{mmol} / \mathrm{L})$ & 5.57 & 5.03 & 5.27 & 1.379 & 0.267 \\
\hline $\lg (\mathrm{FBG})$ & 0.73 & 0.70 & 0.72 & 32.360 & 0.289 \\
\hline FINS(mmol/L) & $29.36^{* *}$ & $18.05^{* *}$ & 5.62 & 32.237 & $<0.001$ \\
\hline HOMA-IR & $7.36^{* *}$ & $4.20^{* *}$ & 1.34 & 25.565 & $<0.001$ \\
\hline $\lg$ (HOMA-IR) & $0.72^{* * \#}$ & $0.50^{* *}$ & 0.04 & 24.075 & $<0.001$ \\
\hline $\mathrm{TG}(\mathrm{mmol} / \mathrm{L})$ & 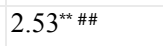 & $1.44^{* *}$ & 0.95 & 27.587 & $<0.001$ \\
\hline $\mathrm{TC}(\mathrm{mmol} / \mathrm{L})$ & 4.40 & 4.42 & 3.88 & 27.587 & 0.127 \\
\hline $\mathrm{HDL}-\mathrm{C}(\mathrm{mmol} / \mathrm{L})$ & $0.94^{* * \# \#}$ & $1.29^{* *}$ & 1.54 & 37.089 & $<0.001$ \\
\hline LDL-C(mmol/L) & $2.94^{* *}$ & $2.72^{* *}$ & 2.05 & 9.566 & $<0.001$ \\
\hline Fatty liver(\%) & $78.26^{* *}$ & $58.73^{* *}$ & 0.00 & 31.242 & $<0.001$ \\
\hline $\operatorname{ALT}(\mathrm{mmol} / \mathrm{L})$ & $64.87^{\text {** }}$ & $61.17^{* *}$ & 14.95 & 45.136 & $<0.001$ \\
\hline $\mathrm{AST}(\mathrm{mmol} / \mathrm{L})$ & $39.83^{\text {** }}$ & $41.03^{* *}$ & 24.41 & 50.953 & 0.001 \\
\hline IL-6(pg/ml) & 5.06 & 4.32 & 4.26 & 1.982 & 0.143 \\
\hline $\mathrm{TNF}(\mathrm{pg} / \mathrm{ml})$ & 26.30 & 26.03 & 23.55 & 0.071 & 0.931 \\
\hline $\lg (\mathrm{TNF})$ & 1.34 & 1.29 & 1.35 & 0.548 & 0.580 \\
\hline mean IMT(mm) & $0.69^{* *}$ & $0.66^{* *}$ & 0.38 & 67.970 & $<0.001$ \\
\hline
\end{tabular}

$\mathrm{BMI}=$ body mass index $; \mathrm{WC}=$ waist circumference; $\mathrm{WHR}=$ waist to hip ratio; $\mathrm{SBP}=$ systolic blood pressure; $\mathrm{DBP}=$ diastolic blood pressure; FBG = fasting blood glucose; FINS = fasting insulin; HOMA-IR = homoeostasis model assessment- insulin resistance; $\mathrm{TG}=$ triglyceride; $\mathrm{TC}=$ total cholesterol; HDL-C = high-density lipoprotein- cholesterol; LDL-C = low-density lipoprotein-cholesterol; ALT = alanine aminotransferase; AST = aspartate aminotransferase; $\lg =$ logarithmical transformation; Compared to control group, ${ }^{* *} P<0.01,{ }^{*} P<0.05$; Compared to obese group, $\# P<0.01, \# P<0.05$. 
Table 2 Correlation between mean IMT and all other variables

\begin{tabular}{|c|c|c|}
\hline \multirow[t]{2}{*}{ Variable } & \multicolumn{2}{|c|}{ Mean IMT } \\
\hline & $\mathrm{r}$ & $\mathrm{p}$ \\
\hline Age(y) & 0.09 & 0.364 \\
\hline Sex & 0.01 & 0.935 \\
\hline Weight(kg) & 0.63 & $<0.001$ \\
\hline $\mathrm{BMI}\left(\mathrm{kg} / \mathrm{m}^{2}\right)$ & 0.68 & $<0.001$ \\
\hline $\mathrm{WC}(\mathrm{cm})$ & 0.70 & $<0.001$ \\
\hline $\lg (\mathrm{WHR})$ & 0.64 & $<0.001$ \\
\hline $\mathrm{SBP}(\mathrm{mmHg})$ & 0.27 & 0.006 \\
\hline $\mathrm{DBP}(\mathrm{mmHg})$ & 0.21 & 0.033 \\
\hline $\lg (\mathrm{FBG})$ & -0.01 & 0.944 \\
\hline FINS(mmol/L) & 0.39 & $<0.001$ \\
\hline $\lg$ (HOMA-IR) & 0.58 & $<0.001$ \\
\hline $\mathrm{TG}(\mathrm{mmol} / \mathrm{L})$ & 0.41 & $<0.001$ \\
\hline $\mathrm{TC}(\mathrm{mmol} / \mathrm{L})$ & 0.17 & 0.080 \\
\hline HDL-C(mmol/L) & -0.41 & $<0.001$ \\
\hline LDL-C(mmol/L) & 0.28 & 0.004 \\
\hline Fatty liver & 0.35 & $<0.001$ \\
\hline $\mathrm{ALT}(\mathrm{mmol} / \mathrm{L})$ & 0.35 & $<0.001$ \\
\hline AST(mmol/L) & 0.30 & 0.002 \\
\hline IL-6(pg/ml) & 0.17 & 0.082 \\
\hline $\lg (\mathrm{TNF})$ & 0.03 & 0.780 \\
\hline
\end{tabular}

$\mathrm{BMI}=$ body mass index; $\mathrm{WC}=$ waist circumference; $\mathrm{WHR}=$ waist to hip ratio; $\mathrm{SBP}=$ systolic blood pressure; $\mathrm{DBP}=$ diastolic blood pressure; $\mathrm{FBG}=$ fasting blood glucose; FINS = fasting insulin; HOMA-IR = homoeostasis model assessment- insulin resistance; TG = triglyceride; TC = total cholesterol; HDL-C = high-density lipoprotein- cholesterol; LDL-C = low-density lipoprotein-cholesterol; ALT = alanine aminotransferase; $\mathrm{AST}=$ aspartate aminotransferase; $\lg =$ logarithmical transformation.

Table 3 Multiple stepwise linear regression analysis, with mean IMT of CCA as the dependent variable and all other variables investigated as the independent variable in all subjects

\begin{tabular}{lllll|}
\hline & $\begin{array}{l}\text { Regression } \\
\text { Coefficient }\end{array}$ & $\begin{array}{l}\text { Standardized } \\
\text { Coefficient }\end{array}$ & $\begin{array}{l}\text { 95\% Confidence } \\
\text { interval }\end{array}$ & $P$ \\
\hline $\mathrm{WC}(\mathrm{cm})$ & 0.003 & 0.292 & $0.001 \sim 0.005$ & 0.009 \\
$\lg (\mathrm{WHR})$ & 0.666 & 0.221 & $0.077 \sim 1.255$ & 0.027 \\
\hline $\mathrm{TG}(\mathrm{mmol} / \mathrm{L})$ & 0.038 & 0.222 & $0.014 \sim 0.062$ & 0.002 \\
\hline $\lg ($ HOMA-IR $)$ & 0.257 & 0.662 & $0.149 \sim 0.366$ & $<0.001$ \\
\hline
\end{tabular}

$\mathrm{WC}=$ waist circumference; $\mathrm{WHR}=$ waist to hip ratio; $\mathrm{TG}=$ triglyceride $;$ HOMA-IR $=$ homoeostasis model assessment- insulin resistance; $\mathrm{lg}=$ logarithmical transformation.

\section{Discussion}

IMT is a well-known marker of subclinical atheroscerosis and it also can indicate future cardio-cerebrovascular disease $[8,12,13]$. Recent reports indicate that the presence of obesity in childhood is associated with increased adult IMT [2,3]. In our study we measured the IMT in obese and nonobese subjects. We found that IMT in obese children and adolescents was significantly increased as compared with non obese children of similar age and sex, which was in accordance with other studies $[14,15,16]$. This tendency was further intensified in the presence of MetS. IMT was closely associated with obesity especially abdominal obesity in childhood and adolescence as confirmed by our correlation analysis and regression analysis.

Obesity has been demonstrated to be associated with cardiovascular risk factors, such as hypertension, dyslipidemia, impaired glucose metabolism and chronic inflammation not only in adults but also in children and adolescents. In our study, IMT was significantly related to $\lg$ (HOMA-IR) and TG in both bivariate correlation and multiple stepwise linear regression analysis, suggesting a link between IMT, insulin resistance and dyslipidemia.

Insulin resistance is a common phenomenon and plays an important role in the cardio-cerebrovascular disease in obese population $[17,18]$. In our study, the obese and MetS group both demonstrated increased fasting insulin than control group rather than fasting blood glucose. Meanwhile, fasting insulin and HOMA-IR levels were significantly related to IMT, however, fasting blood glucose was not related. This information demonstrates that an increased insulin levels seem to be an earlier predictor for atherogenic changes than hyperglycemia, and concur with data published by Atabek et al [19]. Insulin not only di- 
rectly stimulates the expression of vascular cell adhesion molecule [20], but disrupts the balance between the production of NO and ET-1 leading to endothelial dysfunction [21]. Our regression analysis showed that $\lg$ (HOMA-IR) was an independent determinant of mean IMT level, which indicates that insulin resistance was involved in the basic pathological changes associated of obesity [22], and was closely related to cardio-cerebrovascular disease.

Dyslipidemia, especially low HDL-C and high LDL-C, or a high TG is related to cardio-cerebrovascular disease $[23,24]$. These risk factors association with IMT was also shown in our study. According to Pearson correlation analysis, HDL-C, LDL-C and TG were all related to IMT. Therefore, dyslipidemia and cardio-cerebrovascular disease were inseparable. In addition, prevalence of nonalcoholic fatty liver in obese subjects with and without MetS was $78.26 \%, 58.73 \%$ respectively. In contrast, non obese children and adolescents had no fatty liver disease. The correlation between the fatty liver and IMT was significant. It was shown that nonalcoholic fatty liver disease (NAFLD) patients had an increase IMT compared with control subjects in children, just as many other studies have reported $[25,26,27]$.

Deficiencies still exist in our study. First, our sample size was not large enough, especially the number of MetS group. The levels of SBP, DBP, IL-6 and TNF were not statistically related to IMT as other research $[4,5,28,29,30]$. However, the trend of increase was noted. This bias might due to the small sample size. Second, we used the standard of WC in Beijing rather than Zhe Jiang province, which might influence samples selection. Finally, the IMT may also probably be influenced by other risk factors which have not been tested in our study.

In conclusion, atherosclerosis begins in obese children and adolescents, and this tendency is intensified in the presence of MetS. Obesity especially abdominal obesity, high TG level and insulin resistance are strong predictors of increased IMT.

\section{Acknowledgments}

We thank all children and their parents for participating in this research project. We also thank $\mathrm{Li}$ LIANG, Ke HUANG, Jun Fen FU, Xiu Qin CHEN, Fang HONG, Guan Ping DONG, Chun Lin WANG, and Li Qin CHEN for their exceptional patient care and organization. This work was supported, in part, by grant of Zhejiang Science and Technology Agency (2008C03002-1) and Zhejiang Major Medical and Health Science and Technology \& Ministry of Health (WKJ2008-2-026).

\section{Conflict of Interest}

The authors have declared that no conflict of interest exists.

\section{References}

1. Davis PH, Dawson JD, Riley WA, et al. Carotid intimal-medial thickness is related to cardiovascular risk factors measured from childhood through middle age: The Muscatine Study. Circulation. 2001; 104: 2815-9.

2. Raitakari OT, Juonala M, Kähönen $\mathrm{M}$, et al. Cardiovascular risk factors in childhood and carotid artery intima-media thickness in adulthood: the Cardiovascular Risk in Young Finns Study. JAMA. 2003; 290: 2277-83.

3. Li S, Chen W, Srinivasan SR, Bond MG, et al. Childhood cradiovascular risk factors and carotid vascular changes in adulthood the Bogalusa Heart Study. JAMA. 2003; 290:2271-6.

4. Plomgaard P, Bouzakri K, Krogh-Madsen R, et al. Tumor necrosis factor-alpha induces skeletal muscle insulin resistance in healthy human subjects via inhibition of Akt substrate 160 phosphorylation. Diabetes. 2005; 54:2939 -2945.

5. Fain JN. Release of interleukins and other inflammatory cytokines by human adipose tissue is enhanced in obesity and primarily due to the nonfat cells. Vitam Horm.2006; 74:443 -477

6. Suh M, Lee JY, Ahn SV, et al. C-reactive protein and carotid intima-media thickness in a population of middle-aged Koreans. J Prev Med Public Health. 2009; 42:29-34.

7. Csiba L. Carotid intima-media thickness measured by ultrasonography: effect of different pharmacotherapies on atherosclerosis progression. Orv Hetil. 2005; 146: 1239-44.

8. Lorenz MW, Markus HS, Bots ML, Rosvall M, Sitzer M.Prediction of Clinical Cardiovascular Events With Carotid Intima-Media Thickness: a systematic review and meta-analysis. Circulation. 2007; 115:459-67.

9. Working Group On Obesity In China. A national body mass index (BMI) reference for screening overweight and obesity in Chinese school-age children and adolescents. Zhonghua Liu Xing Bing Xue Za Zhi. 2004; 2: 97-102.

10. Zimmet P, Alberti G, Kaufman F, et al, International Diabetes Federation Task Force on Epidemiology and Prevention of Diabetes. The metabolic syndrome in children and adolescent. Lancet. 2007; 369 (9579): 2059-61.

11. Meng Ling-hui, Mi Jie, Cheng Hong, et al. Using waist circumference and waist-to-height ratio to access central obesity in children and adolescents. Chin J Evid Based Pediatr. 2007, 4:245-52

12. Csiba L. Carotid intima-media thickness measured by ultrasonography: effect of different pharmacotherapies on atherosclerosis progression. Orv Hetil. 2005; 146: 1239-44.

13. Urbina EM, Srinivasan SR, Tang R, et al; Bogalusa Heart Study. Impact of multiple coronary risk factors on the intima-media thickness of different segments of carotid artery in healthy young adults (The Bogalusa Heart Study). Am J Cardiol. 2002; 90:953-8.

14. Gustiene O, Slapikas R, Marcinkeviciene J, et al. Relationship between the metabolic sydrome, endothelial funtion and intima-media thickness in asymptomatic middle-aged individuals. Medicina(Kaunas). 2005; 41: 825-36.

15. Zhu W, Huang $X$, He J, et al. Arterial intima-media thickening and endothlial dysfuntion in obese Chinese children. Eur J Pediatr 2005; 164: 337-44.

16. Ianuzzi A, Licenziati $M$, Acampora $C$, et al. Increased carotid intima-media thickness and stiffness in obese children. Diabetes Care. 2004; 27:2506-8.

17. Caceres M, Teran CG, Rodriquez S, et al. Prevalence of insulin resistance and its association with metabolic syndrome criteria 
among Bolivian children and adolescents with obesity. BMC pediatr. 2008; 8:31.

18. Danielsson A, Fagerholm S, Ost A, et al. Short-term overeating induces insulin resistance in fat cells in lean human subjects. Mol Med. 2009; 15:228-34.

19. Atabek ME, Pirgon O, Kivrak AS. Evidence for association between insulin resistance and premature carotid atherosclerosis in children obesity. Pediatr Res. 2007; 61:345-9.

20. Kim I, Moon S-O, HOON Kim S, et al. Vascular endothelial growth factor expression of intercellular adhesion molecule 1(ICAM-1), vascular cell adhesion molecule 1(VCAM-1), and E-selectin through nudear factor-kappa $\mathrm{B}$ activation in endothelial cells. J Biol Chem. 2001; 76:7614-20.

21. Pandolfi A, Solini A, Pellegrini G, et al. Selection insulin resistance affecting nitric oxide release but not plasminogen activator inhibitor-1 synthesis in fibroblasts from insulin -resistance individuals. Arterioscler Thromb Vasc Biol. 2005; 25: 2392-7.

22. Velasco-Martínez RM, Jiménez-Cruz A, Higuera Domínguez F, et al. Obesity and insulin resistance among adolescents from Chiapas. Nutr Hosp. 2009; 24:187-92.

23. Magnussen CG, Venn A, Thomson R, et al. The association of pediatric low- and high-density lipoprotein cholesterol dyslipidemia classifications and change in dyslipidemia status with carotid intima-media thickness in adulthood evidence from the cardiovascular risk in Young Finns study, the Bogalusa Heart study, and the CDAH (Childhood Determinants of Adult Health) study. J Am Coll Cardiol. 2009; 53:860-9.

24. Amarenco P, Labreuche J, Touboul PJ. High-density lipoprotein-cholesterol and risk of stroke and carotid atherosclerosis: a systematic review. Atherosclerosis. 2008; 196:489-96.

25. Pacifico L, Cantisani V, Ricci P, et al. Nonalcoholic fatty liver disease and carotid atherosclerosis in children. Pediatr Res. 2008; 63:423-7.

26. Choi SY, Kim D, Kang JH, et al. Nonalcoholic fatty liver disease as a risk factor of cardiovascular disease: relation of nonalcoholic fatty liver disease to carotid atherosclerosis. Korean J Hepatol. 2008; 14:77-88.

27. Villanova N, Moscatiello S, Ramilli S, et al. Endothelial dysfunction and cardiovascular risk profile in nonalcoholic fatty liver disease. Hepatology. 2005; 42:473-80.

28. Krogh-Madsen R, Plomgaard P, Moller K, et al. Influence of TNF-alpha and IL-6 infusions on insulin sensitivity and expression of IL-18 in humans. Am J Physiol Endocrinol Metab. 2006; 291:E108 -E114.

29. Reinehr T, Kiess W, de Sousa G, et al. Intima media thickness in children obesity relations to inflammatory marker, glucose metabolism, and blood pressure. Metabolism. 2006; 55:113-8.

30. Zanchetti A,Crepaldi G, Bond MG, et al. Systolic and pulse blood pressures (but not diastolic blood pressure and serum cholesterol) are associated with alterations in carotid intima-media thickness in the moderately hypercholesterolaemic hypertensive patients of the Plaque Hypertension Lipid Lowering Italian Study. PHYLLIS study group. J Hypertens. 2001; 19:79-88. 